\title{
Usefulness of measurement of heart rate variability by holter ECG in hemodialysis patients
}

\author{
Nanami Kida ${ }^{1,2^{*}} \mathbb{D}$, Yoshiharu Tsubakihara ${ }^{3}$, Hirota Kida ${ }^{4}$, Shunro Ageta ${ }^{2}$, Makoto Arai ${ }^{5}$, Yoshinosuke Hamada ${ }^{1}$ \\ and Nariaki Matsuura ${ }^{1}$
}

\begin{abstract}
Background: Major adverse cardiac and cerebrovascular event (MACCE) is one of most common complications of hemodialysis patients. Heart rate variability (HRV) is the predictor of death in heart disease patients. However, there are no studies on the role of HRV in hemodialysis patients.

Methods: From September 2009 to March 2011, 24-h electrocardiography was performed in 101 hemodialysis patients. Standard deviation of sequential 5-minute N-N interval means (SDANN) and standard deviation of the N-N interval (SDNN) was examined by a 24-h ECG analysis. Patients were observed prospectively. The primary endpoints were incidence of MACCE and MACCE-free survival.

Results: We studied 90 hemodialysis patients ( 64 males, $63.4 \pm 11.8$ years old). During a follow-up period of $32.0 \pm$ 11.7 months, 33 patients developed MACCE. 24-h ECG showed mean SDNN $93.4 \pm 33.4$ ms and mean SDANN 83.2 $\pm 31.3 \mathrm{~ms}$. MACCE group showed significantly lower SDNN and SDANN than event-free group. In Kaplan-Meier analysis higher SDNN and SDANN group showed significantly higher event-free survival rate than lower group. Using a Cox proportional hazards model, SDNN was independent prognostic factor while SDANN or diabetic status was not significant. In diabetic cases, there were no differences in any factors for the incidence of MACCE between higher SDNN, SDANN groups and lower groups. On the other hand in non-diabetic cases, lower SDNN or SDANN group developed significantly higher MACCE than higher groups.
\end{abstract}

Conclusion: Measurement of HRV by Holter ECG is useful to predict MACCE in hemodialysis patients, especially non-diabetic group.

Keywords: Heart rate variability, Major adverse cardiac and cerebrovascular events, Hemodialysis patients

\section{Background}

Patients on hemodialysis frequently have major adverse cardiac and cerebrovascular events (MACCE) which sometimes lead them to death $[1,2]$ and prediction and prevention of MACCE are important for them. Numerous factors have been reported to induce MACCE in hemodialysis patients. Chronic overhydration status in hemodialysis patients may induce structural or functional

\footnotetext{
* Correspondence: nanami@cam.hi-ho.ne.jp

'Department of Molecular Pathology, Osaka University Graduate School of Medicine and Health Science, 1-1 yamadaoka, 565-0871 Suita City, Osaka, Japan

2Department of Blood Purification Center, Nagahara Hospital, 4-3-13 nagatanishi, 577-0016 Higashiosaka City, Osaka, Japan

Full list of author information is available at the end of the article
}

disorders in myocardium, leading to arrhythmia. Electrolyte imbalance and autonomic nerve disorder, sometimes observed in hemodialysis patients, may cause cardiac sudden death. Oxidative stress, inflammation and abnormal calcium or phosphate metabolism, which are specific to hemodialysis patients, may play some roles in atherosclerosis of coronary artery [3-5]. Hypertension, frequently found in hemodialysis patients, is reported to be the most important factor to cerebrovascular disorders [6]. Although detection of high risk group in hemodialysis patients who would develop MACCE is an important issue, there are no reports on appropriate prognostic biomarkers available for MACCE in hemodialysis patients $[7,8]$. Here we propose 
the usefulness of Holter electrocardiogram (ECG) to predict MACCE in hemodialysis patients.

Holter ECG, conducted over a $24-\mathrm{h}$ period, is widely used as non-invasive measures for the detection of cardiac arrhythmia which is not found in usual ECG. Holter ECG can also evaluate heartbeat change, such as heart rate variability (HRV), by measuring palmic fluctuation. HRV is an index of autonomic activity and includes the standard deviation of the $\mathrm{N}-\mathrm{N}$ interval (SDNN) and standard deviation of sequential 5-minute $\mathrm{N}-\mathrm{N}$ interval means (SDANN). SDNN estimates the standard deviation of the N-N intervals over $24 \mathrm{~h}$, while SDANN reflects the standard deviation of the mean N-N intervals calculated in 5-min segments over $24-\mathrm{h}$. The N-N interval shows the R-R interval between consecutive QRS complex peaks during normal sinus rhythm. Larger fluctuation in the R-R intervals, thus higher HRV, can be found at rest in healthy individuals since heart beats can be physiologically changed by the influence of sympathetic and parasympathetic function according to circadian rhythm. On the other hand, R-R intervals become relatively stable and lower HRV is detected under strong stress or autonomic nerve dysfunction.

HRV is greatly influenced by age and heart rate [9]. Furthermore previous study suggested that patients with diabetes or hypertrophic cardiomyopathy showed decreased HRV [9-13]. It is also reported that the cardiac patients with decreased HRV showed poor prognosis $[14,15]$. Lower HRV is reported to be associated with sudden cardiac death from cardiac arrhythmia or post myocardial infarction [16, 17]. In hemodialysis patients HRV is reduced and decreased HRV is observed already at the stage of chronic kidney failure prior to initiation of dialysis $[18,19]$. However there are no studies on the role of HRV in the prognosis of hemodialysis patients. In this study, we examined the association between HRV and MACCE in hemodialysis patients to determine prognostic factors and the influence of diabetic status on HRV was also studied.

\section{Methods}

From September 2009 to March 2011, 24-h ECG was performed in 101 patients on hemodialysis treated at Osaka General Medical Center, Yuseikai Clinic and Nagahara Hospital. Holter monitor (FM8800; Fukuda Denshi Co Ltd, Tokyo, Japan), was attached to the patients before the start of hemodialysis and data were collected on 2 channels for $24 \mathrm{~h}$. SDANN and SDNN as an index of HRV were examined as well as the check of atrial and ventricular arrhythmias by a 24-h electrocardiographic analysis using an SCM-6600 System (Fukuda Denshi Co, Ltd, Tokyo, Japan).

Clinical data were utilized, including age, gender, body mass index (BMI), duration of hemodialysis, medication of antihypertensives, blood pressure, and fluid removal volume. Biochemical analysis was performed before and after hemodialysis, including serum sodium, potassium, calcium, chloride, blood urea nitrogen, creatinine, albumin, and hemoglobin levels. All the patients did not undergo hemodiafiltration and acetate free biofiltration, but underwent hemodialysis with bicarbonate dialysate and high-flux polysulfone, ethylene vinyl alcohol, and polyethersulfone membrane with a surface area ranging from 0.8 to $2.5 \mathrm{~m}^{2}$.

The hemodialysis patients were examined prospectively for observational study until December 31, 2013. The primary endpoints were incidence of MACCE and MECCE-free survival. The patients were divided into low-level (SDNN 45 cases, SDANN 44 cases) and highlevel groups (SDNN 45 cases, SDANN 46 cases) respectively according to the median value for cutoff line, and all-cause death and MACCE during the observation period were checked.

Subjective 101 patients included 36 females and 65 males. One patient with chronic atrial fibrillation was also excluded because HRV could not be obtained. Ten patients who died of cancer or complication after renal transplantation during the observation period were excluded from the study. Therefore 90 patients (26 females and 64 males) were studied during the observation period.

Informed consent was obtained from all study participants in accordance with the Helsinki Declaration, and the study was approved by the ethical review boards of the treating institutions. Data are shown as mean \pm standard deviation (SD) and statistical analysis was conducted using IBM SPSS software (IBM SPSS version 11.0; IBM, Chicago, Illinois, USA). Continuous variables were compared using a paired t-test, and data of the nominal scale were analyzed by the $x^{2}$ test. Event-free survival data are depicted in a Kaplan-Meier analysis. Univariate and multivariate analyses were conducted to determine the risk factors. Significance level for the primary endpoint was set at $5 \%$.

\section{Results}

Ninety patients (26 females and 64 males) were studied during the observation period; baseline clinical characteristics and biochemical data are shown in Table 1. Mean age was $63.4 \pm 11.8$ years, with mean $68.4 \pm$ 73.4 months duration on hemodialysis. For primary cause of hemodialysis, 44 patients $(48.9 \%)$ had diabetic nephropathy, 30 patients (33.3\%) had chronic glomerulonephritis, and 16 (17.8\%) had unknown or other diseases. Mean BMI was $21.6 \pm 3.2 \mathrm{~kg} / \mathrm{m} 2$. Forty-four patients were treated with angiotensin receptor blockers, 39 with beta blockers, 48 with calcium channel blockers, and 4 with angiotensin-converting enzyme inhibitors. The mean volume of water removed during hemodialysis 
Table 1 Characteristics of the study subjects

\begin{tabular}{|c|c|}
\hline \multicolumn{2}{|l|}{ All patients $(n=90)$} \\
\hline Age (year) & $63.4 \pm 11.8$ \\
\hline Gender (f/m) & $26: 64$ \\
\hline Duration of HD (months) & $68.4 \pm 73.4(1-328)$ \\
\hline \multicolumn{2}{|l|}{ Primary Cause of HD, n (\%) } \\
\hline Diabetic Nephropathy & $44(48.9 \%)$ \\
\hline Chronic glomerulonephritics & $30(33.3 \%)$ \\
\hline Unknown or others & $16(17.8 \%)$ \\
\hline Body Mass Index (kg/m2) & $21.6 \pm 3.2$ \\
\hline PVC,n (\%) & $48(53.3 \%)$ \\
\hline \multicolumn{2}{|l|}{ Medication, n (\%) } \\
\hline ARB & $54(60.0 \%)$ \\
\hline Beta blockers & $39(43.3 \%)$ \\
\hline $48(53.3 \%)$ & Ca channel blockers \\
\hline ACE & $4(4.4 \%)$ \\
\hline Hemoglobin (g/dl) & $10.1 \pm 1.1$ \\
\hline Albumin $(\mathrm{g} / \mathrm{dl})$ & $3.9 \pm 0.5$ \\
\hline pre/postHD Na (mEq/l) & $138.3 \pm 3.5 / 139.9 \pm 2.1$ \\
\hline pre/postHD K (mEq/l) & $4.3 \pm 0.7 / 3.3 \pm 0.4$ \\
\hline pre/postHD Ca (mg/dl) & $9.2 \pm 1.3 / 9.5 \pm 1.0$ \\
\hline Fluid removal (kg) & $2.2 \pm 1.2$ \\
\hline Pre/post HD systolic blood pressure (mmHg) & $153.7 \pm 19.1 / 122.3 \pm 18.2$ \\
\hline Pre/post HD diastolic blood pressure $(\mathrm{mmHg})$ & $70.4 \pm 14.3 / 73.1 \pm 16.6$ \\
\hline
\end{tabular}

$P V C$ premature ventricular contraction, $A R B$ angiotensin receptor blocker, $\mathrm{Ca}$ channel blockers calciumchannel blockers, $A C E$ angiotensin-converting enzyme inhibitor

of 3-4 h was $2.2 \pm 1.2 \mathrm{~kg}$ and the mean systolic/diastolic blood pressure prior to the hemodialysis was $153.7 \pm$ $19.1 \mathrm{mmHg} / 122.3 \pm 18.2 \mathrm{mmHg}$. Blood biochemistry data before hemodialysis were as follows: hemoglobin (Hb), $10.1 \pm 1.1 \mathrm{~g} / \mathrm{dl}$; albumin, $3.9 \pm 0.5 \mathrm{~g} / \mathrm{dl}$; serum sodium, $138.3 \pm 3.5 \mathrm{mEq} / \mathrm{l}$; and serum potassium, $4.3 \pm$ $0.7 \mathrm{mEq} / \mathrm{l}$.

Twenty-four hour ECG shows mean N-N 805.4 \pm 107.2 ms; mean SDNN 93.4 $\pm 33.4 \mathrm{~ms}$ (median 91.5) and mean SDANN, $83.2 \pm 31.3 \mathrm{~ms}$ (median 79.3). The patients were divided into low-level (SDNN 45 cases, SDANN 44 cases) and high-level groups (SDNN 45 cases, SDANN 46 cases) respectively according to the median value for cutoff line, and all-cause death and MACCE during the observation period were checked. During 24-h Holter ECG 48 patients developed premature ventricular contraction (PVC); 41 cases with lowrisk PVCs and 7 cases with high-risk ones such as couplet PVC and PVC short run.

For the mean observation period of $32.0 \pm 11.7$ months (range: 1-49 months), 33 patients (36.7\%) developed MACCE. Thirteen patients (39.4\%) died during the observation period; cause of death is heart failure in 10 patients, arrhythmia in 2 patients and cerebral bleeding in 1 patient. Seventeen (51.4\%) and 4 (12.1\%) patients developed cardiovascular and cerebrovascular complications respectively.

There were no statistical differences in age, hemodialysis duration, BMI, fluid removal volume, $\mathrm{Hb}$, albumin, electrolytes and administration of antihypertensives between the MACCE group and non-MACCE group. The systolic blood pressure change between preand post-hemodialysis was higher in event group than event-free group (35.3+/-19.9 vs $33.0+/-18.6 \mathrm{mmHg} ; p$ $<0.05)$. Event group included more diabetes mellitus (DM) patients than event-free group (66.7 vs $22.2 \%$; $p<$ $0.01)$. Event group showed much lower SDNN and SDANN than event-free group (SDNN $78.3+/-31.2$ vs $102.1+/-31.7 \mathrm{~ms} ; p=0.00$, SDANN $70.7+/-30.7$ vs 90.2 +/-29.5 ms; $p=0.00$ ) (Table 2).

When we compare low and high SDNN groups in the clinical and biochemical data, there were no statistical differences in age, hemodialysis duration, BMI, administration of antihypertensives, $\mathrm{Hb}$, albumin, fluid removal, blood pressure and blood pressure change except DM; 29 DM cases (55.1\%) in 49 low SDNN patients vs 15 DM cases $(36.6 \%)$ in 41 high SDNN ones $(p<0.05)$. However, incidence of MACCE in low SDNN group was

Table 2 Correlated factors with incidence of MACCE

\begin{tabular}{llll}
\hline & $\begin{array}{l}\text { With MACCE } \\
(n=33)\end{array}$ & $\begin{array}{l}\text { Without MACCE } \\
(n=57)\end{array}$ & $P$ value \\
\hline Age (year) & $63.7 \pm 11.9$ & $63.3 \pm 11.8$ & 0.87 \\
HD duration (mounts) & $64.7 \pm 68.9$ & $70.9 \pm 76.1$ & 0.69 \\
DM (n,\%) & $22(66.7)$ & $11(33.3)$ & 0.01 \\
BMl (kg/m2) & $21.3 \pm 3.5$ & $21.8 \pm 3.0$ & 0.49 \\
PVC (n,\%) & $26(78.8)$ & $7(21.2)$ & 0.01 \\
Hb (g/dl) & $10.4 \pm 1.0$ & $10.5 \pm 1.2$ & 0.78 \\
Alb (g/dl) & $3.8 \pm 0.4$ & $3.9 \pm 0.5$ & 0.50 \\
Fluid removal (kg) & $2.3 \pm 1.3$ & $2.2 \pm 1.2$ & 0.51 \\
ARB (n,\%) & $16(48.5)$ & $23(40.4)$ & 0.45 \\
$\beta$ B blocker (n,\%) & $19(57.6)$ & $35(61.4)$ & 0.72 \\
Ca channel blockers (n,\%) & $17(51.5)$ & $31(54.3)$ & 0.63 \\
Na (mEq/l) & $139 \pm 3.4$ & $138.2 \pm 3.5$ & 0.30 \\
K (mEq/l) & $4.4 \pm 0.8$ & $4.2 \pm 0.7$ & 0.47 \\
Ca (mEq/l) & $9.1 \pm 1.3$ & $9.3 \pm 1.3$ & 0.49 \\
SDNN (ms) & $78.3 \pm 31.2$ & $102.1 \pm 31.7$ & $<0.001$ \\
SDANN (ms) & $70.7 \pm 30.7$ & $90.2 \pm 29.5$ & $<0.001$ \\
$\triangle$ Sys blood pressure (mmHg) & $35.3 \pm 19.9$ & $33.0 \pm 18.6$ & 0.05 \\
$\triangle$ Dia blood pressure (mmHg) & $14.5 \pm 7.8$ & $13.2 \pm 8.1$ & 0.47 \\
\hline DM d $(\mathrm{m}$ (m) & &
\end{tabular}

$D M$ diabetes mellitus, $B M I$ body mass index, $P V C$ premature ventricular contraction, $\mathrm{Hb}$ hemoglobin, Alb albumin, $A R B$ angiotensin receptor blocker, Ca channel blockers calciumchannel blockers, SDNN standard deviation of the $\mathrm{NN}$ interval, SDANN standard deviation of sequential five-minute N-N interval means 
$55.1 \%$ (27/49), much higher than that in high SDNN group, $14.6 \%(6 / 41) \quad(p<0.01)$. Also low and high SDANN groups were compared in the similar ways. There were no statistical differences in any data including DM. However, low SDANN group had much higher incidence of MACCE than high SDANN group (47.4\% $(27 / 57)$ vs $18.2 \%(6 / 33) ; p=0.01)$. Low SDNN group developed PVC more frequently than high SDNN group during 24-h ECG $(p<0.05)$ as well as more frequent incidence of PVC in low SDANN group $(p<0.03)$.

In Kaplan-Meier analysis higher SDNN group showed much higher event-free survival rate than lower SDNN group (Log rank, $p=0.00$ ) as well as higher SDANN group showed significantly higher survival rate (Log rank, $p=0.01$ ) (Fig. 1). In the longer follow-up time the difference of event-free survival between SDNN higher and lower groups, as well as SDANN higher and lower groups, seems to be increased. In the analysis using a Cox proportional hazards model, SDNN was determined to be an independent prognostic factor $(p=0.02$; hazard ratio: 0.57 ) while SDANN or diabetic status was not significant in multivariate analysis (Table 3 ).

Predictive factors for MACCE are analyzed separately in both diabetic and non-diabetic patients. In diabetic 44 cases 22 patients (50\%) developed MACCE and there were no significant differences in any factors including SDNN and SDANN for the incidence of MACCE. On the other hand 11 patients (23.9\%) developed MACCE in non-diabetic 46 cases, much less rate than diabetic group. There were no significant differences in age, hemodialysis duration, hemoglobin level, albumin level, or BMI for MACCE. However, lower SDNN or SDANN group developed significantly higher MACCE than higher groups $(p=0.00$ or $p=0.04$, Table 4$)$.

\section{Discussion}

Although the prognosis of the hemodialysis patients has been improved, they still have higher risk for MACCE and earlier detection of MACCE is important in clinical practice [20]. In hemodialysis facility anemia, nutrition and bone metabolic markers are usually checked by the laboratory examination, but it is difficult for the prediction of MACCE from those laboratory data. Radiography like coronary angio-CT or ultrasonography is not usually so convenient in hemodialysis facility and sufficient examination for early detection is not performed. In this study we investigate the possibility of prediction of prognosis of the hemodialysis patients by Holter ECG, easy and non-invasive measures available in usual hemodialysis facility.

HRV obtained by Holter ECG reflects the balance of sympathetic and parasympathetic nervous function $[9,10]$. Decreased HRV defined by SDNN or SDANN, which shows no difference in heartbeats between in the daytime and at night, is considered as a state of sympathetic hyperactivity. Activated condition of sympathetic nerve function is known to induce lethal arrhythmia leading to sudden death [21]. An earlier study reported that prognosis of patients with heart failure or ischemic heart disease was intimately linked to $\operatorname{HRV}[22,23]$.

In this study the incidence of MACCE was significantly correlated with diabetic status, low SDNN, low SDANN and high blood pressure change during hemodialysis. Our result is consistent with the previous reports in which the hemodialysis patients from diabetic nephropathy have higher risk for MACCE [24]. Our results that the patients with MACCE showed significantly lower SDNN and SDANN were noteworthy because decreased HRV might reflect high cardiac overload
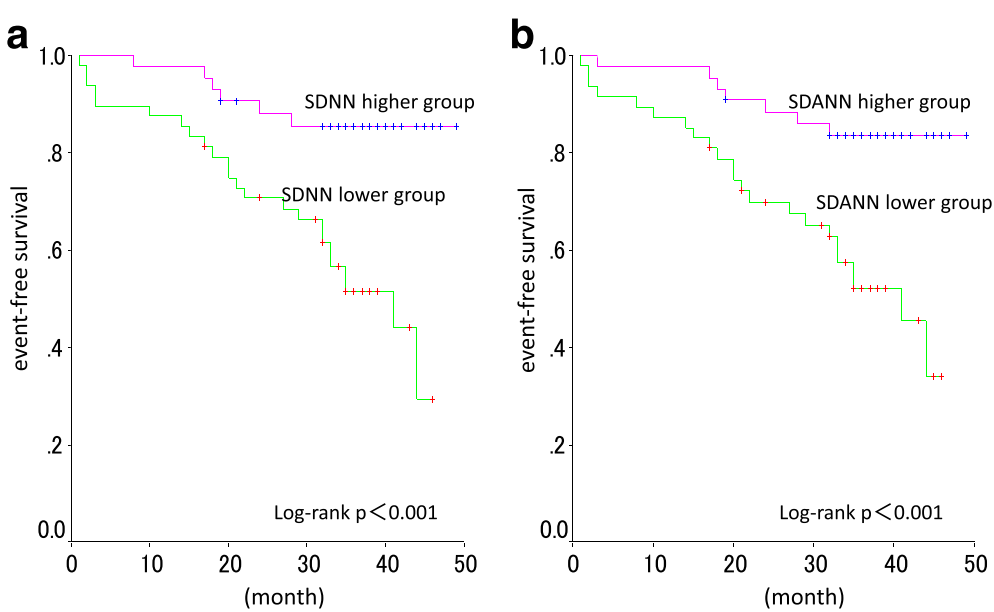

Fig. 1 Event-free survival of hemodialysis patients with higher and lower heart rate variability. captions: a SDNN higher group showed significantly higher MACCE-free survival than SDNN lower group. b SDANN higher group showed significantly higher MACCE-free survival than SDANN lower group 
Table 3 COX regression model for MACCE

\begin{tabular}{|c|c|c|c|c|}
\hline & \multicolumn{2}{|c|}{ Univariate analysis } & \multicolumn{2}{|c|}{ Multivariate analysis } \\
\hline & $p$ value & $\begin{array}{l}\text { Hazard ratio } \\
(95 \% \mathrm{Cl})\end{array}$ & $p$ value & $\begin{array}{l}\text { Hazard ratio } \\
(95 \% \mathrm{Cl})\end{array}$ \\
\hline Age & 0.78 & $1.0(0.98-1.02)$ & & \\
\hline HD duration & 0.84 & $1.0(0.99-1.00)$ & & \\
\hline $\mathrm{DM}$ & 0.02 & $1.6(1.09-2.56)$ & 0.06 & $1.5(0.9-2.4)$ \\
\hline SDNN & 0.01 & $0.5(0.37-0.87)$ & 0.02 & $0.57(0.36-0.89)$ \\
\hline SDANN & 0.12 & $0.7(0.48-1.14)$ & & \\
\hline PVC & 0.06 & $2.1(0.88-2.73)$ & & \\
\hline ARB & 1.00 & $1(0.50-1.99)$ & & \\
\hline$\beta$ blocker & 0.54 & $0.9(0.57-1.34)$ & & \\
\hline $\mathrm{Hb}$ & 0.90 & $1.0(0.82-1.20)$ & & \\
\hline Alb & 0.11 & $1.5(0.91-2.46)$ & & \\
\hline Fluid removal & 0.30 & $1.1(0.92-1.32)$ & & \\
\hline $\begin{array}{l}\triangle \text { Systolic Blood } \\
\text { Pressure }\end{array}$ & 0.20 & $1.0(0.99-1.02)$ & & \\
\hline
\end{tabular}

DM diabetes mellitus, SDNN standard deviation of the NN interval, SDANN standard deviation of sequential five-minute $\mathrm{N}-\mathrm{N}$ interval means, $P V C$ premature ventricular contraction, $A R B$ angiotensin receptor blocker, $\mathrm{Hb}$ hemoglobin, Alb albumin

resulting in MACCE. Higher blood pressure change during hemodialysis significantly associated with MACCE means that cardiac function was so poor that decreased circulating blood volume due to hemodialysis may develop higher blood pressure change.

In this study SDNN and SDANN low-level groups had higher incidence of MACCE and worse outcome than the high-level groups and also SDNN and SDANN lower groups showed significantly lower event-free survival in Kaplan-Meier analysis than higher groups. Furthermore SDNN was an independent prognostic factor in COXhazard analysis while SDANN was not. SDNN estimates the standard deviation of the mean values of $\mathrm{NN}$ interval for $24 \mathrm{~h}$ and reflects an HRV change over the long-term more strongly compared with SDANN which indicates the standard deviation of the mean N-N intervals calculated in 5-min segments over 24-h. Therefore, SDNN is a more sensitive predictor of prognosis than SDANN.
These results suggest that body fluid volume should be strictly regulated by hemodialysis and that early detection of cardiovascular complications should be recommended in the hemodialysis patients with HRV decrease since those patients were at high risk for MACCE. When we analyzed incidence of PVC, low SDNN group developed PVC more frequently as well as low SDANN group. PVC has been reported to be associated with age, sympathetic nerve imbalance and cardiac dysfunction. Furthermore, the patients with PVC had more incidence of MACCE $(p<0.01)$, suggesting low SDNN and SDANN might result ventricular arryhtmia and that could be predictable factors for MACCE .

In the current study HRV was not correlated with age or dialysis history. Our result might suggest that some patients showed activated sympathetic function resulting in poor prognosis already at the early stage of chronic renal failure. Generally long term dialysis is supposed to induce MACCE. However, there are high risk group of hemodialysis patients even at the initiation stage of hemodialysis with the increased older population or diabetic status for hemodialysis. As previous study showed HRV are already decreased at the stage of advanced chronic kidney disease prior to initiation of dialysis, the measurement of HRV at the initiation of hemodialysis would be useful for the detection of high risk group of MACCE [18]. In this study there were no differences in SDNN and SDANN between no medications and medications with of beta blocker or calcium blocker. Previous study reported medication with calcium blocker induced activation of parasympathetic function, resulting in short-term HRV such as mean R-R intervals calculated in 5-min segments [25]. In this study we tested only long-term HRV and so no differences were observed.

The hemodialysis patients with diabetic nephropathy as primary disease have been reported to show poor prognosis. In this study low SDNN or SDANN group showed similar incidence of MACCE to high SDNN or SDANN group in the diabetic patients, whereas low SDDN or SDANN group demonstrated significantly higher incidence of MACCE than high group in non-

Table 4 MACCE-correlated factors in diabetic and non-diabetic subjects

\begin{tabular}{|c|c|c|c|c|c|c|}
\hline \multicolumn{4}{|l|}{$\mathrm{DM}(n=44)$} & \multicolumn{3}{|l|}{ Non-DM $(n=46)$} \\
\hline & With MACCE $(n=22)$ & Without MACCE $(n=22)$ & $p$ value & With MACCE $(n=11)$ & Without MACCE $(n=35)$ & $p$ value \\
\hline Age (year) & $61.9 \pm 12.8$ & $63.1 \pm 12.6$ & 0.75 & $67.4 \pm 9.6$ & $63.4 \pm 11.4$ & 0.41 \\
\hline HD duration (months) & $46.0 \pm 11.6$ & $46.6 \pm 10.5$ & 0.97 & $102.1 \pm 81.8$ & $86.3 \pm 86.1$ & 0.83 \\
\hline Alb (g/dl) & $3.9 \pm 0.4$ & $4.0 \pm 0.5$ & 0.60 & $3.7 \pm 0.4$ & $3.9 \pm 0.5$ & 0.86 \\
\hline $\mathrm{Hb}(\mathrm{g} / \mathrm{dl})$ & $10.5 \pm 1.1$ & $10.7 \pm 1.0$ & 0.61 & $10.3 \pm 0.8$ & $10.4 \pm 1.3$ & 0.81 \\
\hline Fluid removal (kg) & $2.4 \pm 1.5$ & $2.1 \pm 1.1$ & 0.41 & $2.2 \pm 1.1$ & $2.2 \pm 1.2$ & 0.91 \\
\hline SDNN (Low,n\%) & $16(72.7 \%)$ & $11(50.0 \%)$ & 0.12 & $7(63.6 \%)$ & $11(31.4 \%)$ & 0.05 \\
\hline SDANN (Low,n\%) & $16(72.7 \%)$ & $13(59.1 \%)$ & 0.34 & $8(72.7 \%)$ & $9(25.7 \%)$ & 0.01 \\
\hline
\end{tabular}

Alb albumin, $\mathrm{Hb}$ hemoglobin, SDNN standard deviation of the NN interval, SDANN standard deviation of sequential five-minute N-N interval means 
diabetic patients. Earlier research has shown that diabetic condition itself is a strong risk factor for MACCE and the contribution of HRV might be relatively low. On the other hand, low SDNN or SDANN patients show higher incidence for MACCE in non-diabetic patients in our study, suggesting low HRV might be a strong risk factor for prognosis in non-diabetic hemodialysis patients.

There are several limitations to our study. First, the sample size of 90 patients was small. Secondly cardiac function by echocardiography was not evaluated since echocardiogram was not equipped in one clinic and the association of SDNN and SDANN with cardiac function such as left ventricular end-diastolic dimension and left ventricular ejection fraction. Thus the relationship between MACCE and cardiac function was not discussed sufficiently.

\section{Conclusion}

Measurement of HRV by Holter ECG is useful to predict MACCE in hemodialysis patients, especially nondiabetic group.

\section{Abbreviation}

BMI: Body mass index; ECG: Electrocardiogram; Hb: Hemoglobin; HRV: Heart rate variability; MACCE: Major adverse cardiac and cerebrovascular events; PVC: Premature ventricular contraction; SDANN: Standard deviation of sequential 5-minute N-N interval means; SDNN: Standard deviation of the $\mathrm{N}-\mathrm{N}$ interval

\section{Acknowledgements}

This work was supported by making acquisition of data from Osaka general medical center, Yuseikai clinic, and Nagahara hospital of blood purification center, Osaka, Japan.

\section{Funding}

The study was no specific funding.

\section{Availability of data and materials}

No data has been submitted to any open access databases. All data supporting the study is presented in the manuscript or is available upon request.

\section{Authors' contributions}

NK: design, data acquisition, analysis, interpretation, drafting manuscript, final approval. YT: contributions to conception and design, final approval. HK: data acquisition, analysis. SA: contributions to conception and design, data acquisition, final approval. MA: contributions to conception and design, data acquisition, final approval. YH: interpretation of data, final approval. NM: interpretation of data, drafting manuscript, final approval, manuscript revision.

\section{Competing interests}

The authors declare that they have no competing interests.

\section{Consent to publish}

Not applicable.

\section{Ethics approval and consent to participate}

The Ethics Committee of Osaka General Medical Center approved the study protocol, with approval number 21-S0718. Informed written and oral consent was obtained from all participants prior to participation in the study.

\section{Author details}

'Department of Molecular Pathology, Osaka University Graduate School of Medicine and Health Science, 1-1 yamadaoka, 565-0871 Suita City, Osaka, Japan. ${ }^{2}$ Department of Blood Purification Center, Nagahara Hospital, 4-3-13 nagatanishi, 577-0016 Higashiosaka City, Osaka, Japan. ${ }^{3}$ Department of Management in Health Care Sciences, Graduate School of Health Care Science, Jikei Institute, 1-2-8 miyahara yodogawaku, 532-0003 Osaka City, Japan. ${ }^{4}$ Department of Clinical Engineering, Osaka General medical Center, 3-1-56 bandaihigashi sumiyoshiku, 558-0056 Osaka City, Japan. ${ }^{5}$ Department of Internal Medicine, Yuseikai Clinic, 10-39 hideincyo tennojiku, 543-0055 Osaka City, Japan.

Received: 4 June 2016 Accepted: 20 December 2016 Published online: 05 January 2017

References

1. K/DOQI Workgroup. K/DOQI clinical practice guidelines for cardiovascular disease in dialysis patients. Am J Kidney Dis. 2005;45:S1-153.

2. Omotoso BA, Abdel-Rahman EM, Xin W, Ma JZ, Scully KW, Aroqundade FA, et al. Acute kidney injury(AKI) outcome, a predictor of long-term major adverse cardiovascular events(MACE). Clin Nephrol. 2016;85:1-11.

3. Lee MJ, Doh FM, Kim CH, Koo HM, Oh HJ, Park JT, et al. Interdialytic weight gain and cardiovascular outcome in incident hemodialysis patients. Am J Nephrol. 2014:39:427-35.

4. Yasuaki D, Masui O, Masaya S, Hiroyuki T, Koichi S, Ryuzo U. Candesartan reduces oxidative stress and inflammation in patients with essential hypertension. Hypertens Res. 2003;26:691-7

5. Fabbian F, Cacici G, De Biase V, Yabarek T, Gangemi C, Franceschini L, et al. Relationship between major adverse cardiac events and angiographic findings in dialysis patients. Int Urol Nephrol. 2011;43:1171-8.

6. Foley RN, Parfrey PS, Harnett JD, Kent GM, Murray DC, Barre PE. Impact of hypertension on cardiomyopathy, morbidity and mortality in end-stage renal disease. Kidney Int. 1996:49:1379-85.

7. Bleyer AJ, Hartman J, Brannon PC, Reeves-Daniel A, Satko SG, Russell G. Characteristics of sudden cardiac death in haemodialysis patients. Kidney Int. 2006;69:2268-73.

8. Herzog CA, Mangrum JM, Passman R. Sudden cardiac death and dialysis patients. Semin Dial. 2008;21:300-7.

9. Task Force of European Society of Cardiology and The North American Society of Pacing and Electrophysiology. Heart rate variability; Standard of measurement, physiological interpretation, and clinical use. Eur Heart J. 1996:17:354-81.

10. Junichiro H, Andrew T, Seiji M, Akiyoshi O, Yosaku W, Kazuyuki T, et al. Assessment of frequency shift in R-R interval variability and respiration with complex demodulation. Am Phys Soc. 1994;77:2879-88.

11. Willich SN, Levy D, Rocco MB, Tofler G, Stone PH, Muller JE. Circadian variation in the incidence of sudden cardiac death in the Framingham Heart Study population. Am J Cardiol. 1987:60:801-6.

12. Furlan R, Guzzetti S, Crivellaro W, Dassi S, Tinelli M, Baselli G, et al. Continuous 24-hour assessment of the neural regulation of systemic arterial pressure and RR variabilities in ambulant subject. Circulation. 1990;81:537-47.

13. Wan-tai M, Per GR, Jeanne EP, Jill A, George J, Ross DF, et al. SCD-HeFT: use of $R-R$ interval statistics for long-term risk stratification for arrhythmic sudden cardiac death. Heart Rhythm. 2015:12:2058-66.

14. Schwartz PJ, La Rovere MT, Vanoli E. Autonomic nervous system and sudden cardiac death. Experimental basis and clinical observations for postmyocardial infarction risk stratification. Circulation. 1992;85:177-91.

15. Nolan J, Batin PD, Andrews R, Lindsay SJ, Brooksby P, Mullen M, et al. Prospective study of heart rate variability and mortality in chronic heart failure; results of United Kingdom Heart Failure Evaluation and Assessment of Risk Trial(UK-Heart). Circulation. 1998;98:1510-6.

16. Reena R, Martica H, Chris TC, Mark U. Heart Rate Variability(HRV) in kidney failure: measurement and consequences of reduced HRV. Nephrol Dial Transplant. 2008;23:444-9.

17. Daniel JB, Lori DB, Rehan Q, Deidra C, Eric AW, Brad CA, et al. Heart rate variability predicts ESRD and CKD-related hospitalization. J Am Soc Nephrol. 2010:21:1560-70.

18. Hase H, Tsunoda T, Tanaka Y, Takahashi Y, Imamura Y, Ichikawa H, et al. Risk factors for de novo acute cardiac events in patients initiating hemodialysis with no previous cardiac symptom. Kidney Int. 2006;70:1142-8. 
19. Bigger JT, Fleiss JL, Steinman RC, Rolnitzky LM, Kleiger RE, Rottman JN. Frequency domain measures of heart period variability and mortality after myocardial infarction. Circulation. 1992;85:164-71.

20. Bilchick KC, Fetics B, Djoukeng R, Fisher SG, Fletcher RD, Singh SN, et al. Prognostic value of heart rate variability in chronic congestive heart failure (Veterans Affaies' Survival Trail of Antiarrhythmic Therapy in Congestive Heart Failure). Am J Cardiol. 2002;90:24-8.

21. Kleiger RE, Miller P, Bigger JT, Moss AJ. Decreased heart rate variability and its association with increased mortality after acute myocardial infarction. Am J Cardiol. 1987;59:256-62.

22. Mauro G, Daniela M, Giuseppe P, Alberto C, Michele V, Carmelo G. Differences in heart rate variability parameters during the post-dialytic period in type Ildiabetic and non-diabetic ESRT patients. Nephrol Dial Transplant. 2001;16:566-73.

23. Preeti C, Robin LS, Brenda WG, Nathan WL, Peter K, Margaret K, et al. Predictors of heart rate variability and its prognostic significance in chronic kidney disease. Nephrol Dial Transplant. 2012;27:700-9.

24. Ramirez S, McCullough K, Thumma J, Nelson RG, Morgenstern H, Gillespie BW, et al. Hemoglobin A1c levels and mortality in the diabetic hemodialysis population: findings from the Dialysis Outcomes and Practice Patterns Study (DOPPS). Diabetes Care. 2012;21:2527-32.

25. Purushothaman $P, E$ Sankaranarayanan $P$, Tarun KD, Trakroo M. Effect of antihypertensive drug therapy on short-term heart rate variability in newly diagnosed essential hypertension. Clin Exp Pharmac Physiol. 2010;37:107-13.

\section{Submit your next manuscript to BioMed Central} and we will help you at every step:

- We accept pre-submission inquiries

- Our selector tool helps you to find the most relevant journal

- We provide round the clock customer support

- Convenient online submission

- Thorough peer review

- Inclusion in PubMed and all major indexing services

- Maximum visibility for your research

Submit your manuscript at www.biomedcentral.com/submit 